\title{
BLOCKCHAIN ENABLED IOT SYSTEM ARCHITECTURE IN EDGE COMPUTING ENVIRONMENTS
}

\author{
Nicolae VRANĂ \\ Bucharest University of Economic Studies, Romania \\ vrananicolae17@stud.ase.ro
}

\begin{abstract}
The Internet of Things paradigm enables various applications in different fields through the exponential increase of devices and sensors. Architecting systems based on Internet of Things concepts is very challenging and has focused mostly on a centralized approach. Decentralized or distributed architectures aren't straightforward concepts for IoT applications, but through the distributed ledger and Blockchain implementations that provide better identification, security and data integrity the fully distributed system architecture can become a reality. Major cloud vendors these days are using edge computing platforms to bring the cloud closer to the device, using a locally centralized system based on edge agents, which can be seen as a partially decentralized module in the full IoT cloud picture. Blockchain allows this edge IoT environment to become distributed if services or functions installed on the edge agent are used by the device to gain Blockchain capabilities. If more capable devices are deployed, then the Blockchain functionality can be embedded in the IoT and the system becomes a fully distributed peer-to-peer network.
\end{abstract}

Keywords: Internet of Things, Blockchain, Edge Computing, Serverless

JEL classification: C88, L63, L86

DOI: $10.12948 / \mathrm{ie} 2019.02 .04$

\section{Introduction}

The fourth industrial revolution brings numerous technological advances such as autonomous vehicles, 3D printing, advanced robotics, new materials and it is expected to have 1 trillion sensors connected to the internet by 2025 [1]. In the digital world we are already talking about big data processing techniques, automation, machine learning, distributed ledgers and the internet of things [2].

This article analyses how blockchain can improve the shortcomings and solve the integrity and security problems of current IoT architectures. Due to the capability constraints of IoT devices there can be partially distributed solutions for the less capable or fully distributed architectures for the devices that have higher computational and storage features.

\section{Definitions and technical considerations}

Internet of Things (IoT) is a term first mentioned by Kevin Ashton in 1999 in a presentation at Procter \& Gamble [3 - 4] and it describes a world of devices connected to the internet and to each other. IoT got traction after sensors became cheaper and microcontrollers and microcomputers started to become mainstream.

Blockchain is defined by the Oxford dictionary as "A system in which a record of transactions made in bitcoin or another cryptocurrency are maintained across several computers that are linked in a peer-to-peer network" [5]. A more useful definition for the present study to address a broader context as "A technology that enables immutability, and integrity of data in which a record of transactions made in a system is maintained across several distributed nodes that are linked in a peer-to-peer network" [6]. 
Edge Computing is a term used to bring together Cloud Computing and IoT. Devices generate huge amount of data that needs to be sent to the Cloud to be analyzed, audited and tracked, data traffic that will take much more time than if it will be processed on a machine close to the place the data was created. Cisco issued the term Fog Computing which they wanted to impose as a standard for Edge Computing [7].

Serverless is a cloud computing paradigm that refers to programs that run in stateless compute containers which are event-triggered, only last for one invocation and fully managed [8]. Cloud providers nowadays offer serverless capabilities through managed services such as AWS lambda, Microsoft Azure functions and Google cloud functions.

\section{Centralized IoT architecture}

The big cloud vendors have improved their IoT offerings nowadays and are delivering to their customers edge managed environments or serverless managed services that greatly improve device connectivity, control, authentication, identity, security and data analysis.

The cycle starts with the software or even the operating system running on the device that can seamlessly connect to the edge or cloud services designed to manage the IoT devices fleet seen below in the Control Services phase in Figure 3. The control step is also responsible for managing the protocol jungle (if the devices communicate using different protocols), for transforming the received data into meaningful information that can be transferred to the cloud services specialized in analyzing and machine learning. The analyzed data in the Data Services phase will provide useful insights into the collected information and will help predict outcomes such as when the device is going to malfunction and help taking automatic preemptive action. The cycle doesn't stop after the data is analyzed, but it will use the information and maybe some manual inputs that can be used to feed the device and start the improved cycle all over again.

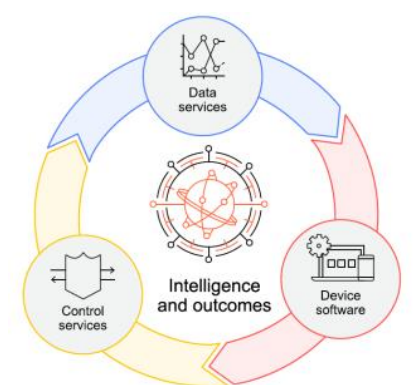

Figure 3 IoT virtuous cycle (Source: AWS Summit Berlin [9])

The traditional centralized IoT architecture depicted in Figure 4 includes the IoT devices and sensors in a secure private network that are communicating to an edge cloud agent that in turn sends and receives data to and from the cloud. In this situation the agent does all the heavy lifting of computation, data storage and traffic. It needs to run locally different serverless functions that will check the integrity of the data, will parse different data formats coming from the devices and will transform it in meaningful information accepted by other services running locally or in the cloud.

The agent needs to identify each IoT device sending a data package and it will need to authenticate itself with the cloud, each package sent has to be encrypted with a local certificate installed on the machine. Certificate rotation is a must if we need to preserve a high security standard especially if the process has a high automation degree and it isn't attended by security professionals. 


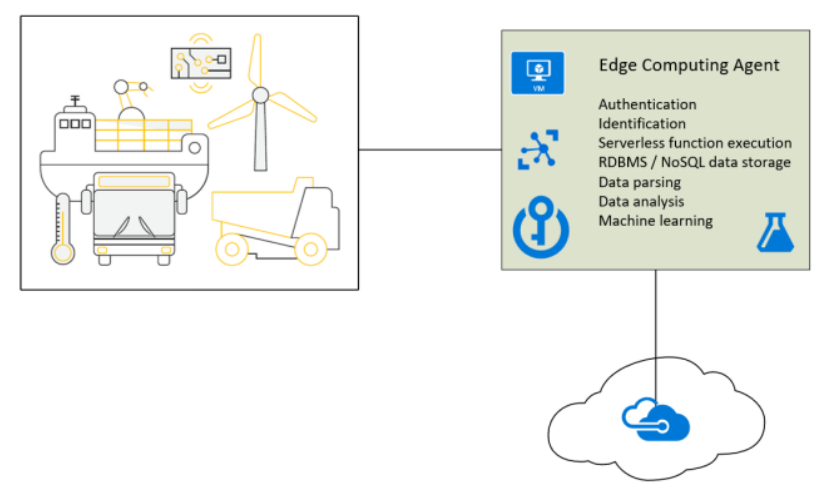

Figure 4 IoT centralized architecture

Shortcomings with the centralized architecture include the single point of failure problem. There is one node in the system that can make everything crash and that is the edge cloud agent. We need to make sure the machine is running every time we need it by replicating it throughout our network. This means each replica needs to run the same critical services as the original and this way we lose the benefits cloud brings such as on-demand computational power.

Scalability is another issue, because when more IoT devices are added the agent will have to cope with more network traffic and more operations for each device.

\section{Security and data integrity}

Security is a huge concern in the IoT world where devices are vulnerable to attacks that permit the attacker to gain control of the entire network through a compromised device [10]. To protect the devices, the network and the private resources in the cloud the agents need to authenticate and identify IoT devices prior to adding them to the system. Also, periodically checking and testing the integrity of the device and the software is necessary [11].

There are several types of attacks depending on the layer and these are: software attacks, denial of service attacks, collected and transferred data attacks, network protocols attacks, communication channels attacks, physical device attacks [12].

In a centralized architecture data resides in few places and poses great vulnerabilities to Denial of Service (DoS) and Distributed Denial of Service (DDoS) attacks where the data source, the IoT device and the cloud or edge server where the data will eventually reside are easy targets [13]. DoS attacks will eventually render useless the devices in a short amount of time due to their limited capabilities that will be depleted. Cloud providers nowadays are well prepared for such attacks on the servers that reside in their farms, but edge machines are more vulnerable and need special attention in a traditional network and security environment. Periodically auditing and interpreting the log data and events occurring on these servers through advanced analytics is a must. Devices can be deactivated when an anomaly occurs and the team in charge can perform automatic tests and manual verifications, update frameworks and rotate credentials.

Data integrity is at risk when a hacker takes control of the device and sends misleading signals or tries to take over the network. Using false data to gain different advantages is also called tampering. Through Blockchain technology we can gain more control on the data being delivered and there are protocols that enable information confidentiality, integrity and authenticity [14].

\section{Distributed IoT architecture}

A distributed system is composed of autonomous elements interconnected in a peer-to-peer network with increased computing and storage capabilities. There are several distributed 
architectures that can be employed for IoT and one of them is the distributed ledger and particularly Blockchain. Blockchain is a distributed and decentralized ledger that generates and stores transactions in blocks that are linked in a time series. Data inside blocks is immutable and hashes containing links to previous blocks are generated [13].

Blockchain brings democracy to IoT systems where all transactions and operations are carried out through consensus. Peer-to-peer communication enables processing of billions of transactions.

\subsection{Blockchain IoT Pseudo-Distributed Architecture}

Due to the fact that most of the IoT devices have limited capabilities they won't be able to perform node like tasks in a Blockchain peer-to-peer network. So, arises the need to mirror the device on the edge computing machine to add Blockchain capabilities as seen in Figure 5.

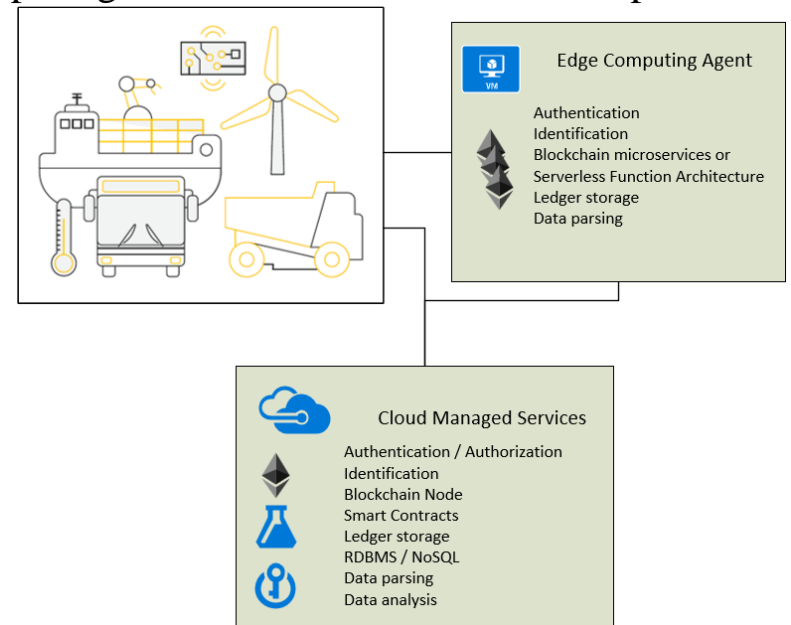

Figure 5Blockchain IoT Pseudo-Distributed Architecture

The IoT device sends a raw signed transaction to the edge agent Blockchain endpoint and this, in turn, creates the cryptographic transaction that will be added to the chained block by mutual consensus with the other nodes. Each blockchain endpoint can reside on the edge agent in a microservice [15] or in a serverless function. The serverless architecture is similar to the centralized one, but this time the function as a service performs Blockchain related operations. The functions are available on demand and they can scale per request directly from the devices or if an event based communication is implemented then the requests can be allocated through an event queue and so the system resources could be better managed.

In case of the microservices architecture the system needs to be up and running and respond to requests in real time. As a proof of concept an orchestration cluster such as Kubernetes can be installed on 3 or more Raspberry Pi Model B single-board computers, on one of them we install the master node and the others will be the worker nodes [16]. The worker nodes will run the Blockchain microservices and will expose the endpoints in REST Api standard.

On the single-board computers we can also install OpenFaas which is a framework for building Serverless functions with Docker and Kubernetes and host them on premise [17].

\subsection{Blockchain IoT Distributed Architecture}

There are situations when the devices have superior computational capabilities and support Blockchain operations inside the microcontroller or the single-board computers installed. Processing the transactions themselves these IoT devices enable our fully distributed architecture as seen in Figure 6 and reduce traffic to and from the edge computing agent [15]. 
www.conferenceie.ase.ro

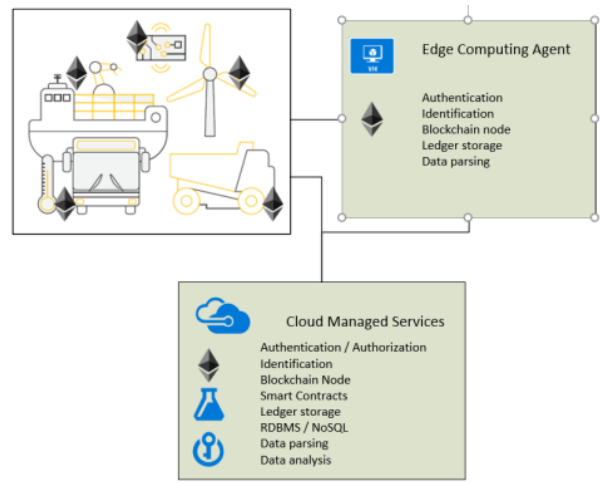

Figure 6 Blockchain IoT Distributed Architecture

Lately, there are several Blockchain implementations that allow fewer amounts of ledger data to be stored on the node. One of these is the simplified payment verification (SPV) which allows transactions to be verified without running block mining operations and without storing all historical blocks [18]. Also, Ethereum has introduced the concept of light client that connects to a more powerful node on an edge agent allowing it to store less data and perform less operations [15].

\section{Further research and development}

Smart contracts are pieces of software that execute upon meeting certain conditions. This is achieved by automatically executing code that is distributed and verified by the decentralized Blockchain network nodes [19]. The concept of smart contracts can further extend the proposed Blockchain IoT architecture by enabling automatic code execution when different events occur, for example when a user pays for the rented car through the mobile application using tokens then the device will automatically unlock the car.

Smart contracts can also be used to execute calls to legacy enterprise systems such as web servers, application servers, databases that cannot be easily migrated to the Blockchain [15]. Smart contracts also come with downsides like possible bugs implemented in the contract or hacking vulnerability [20].

Further developing the architecture, we need to think about the huge amount of data generated by the IoT devices and how this data needs to be optimally stored in the cloud or if it can be discarded as not being useful in the future and how this breaks the Blockchain immutability property. Time series databases and stream analytics combined with machine learning and artificial intelligence managed services can provide more useful insights.

\section{Conclusion}

The distributed Blockchain IoT architecture discussed in this paper presents a different approach to designing and leveraging the IoT solutions in the cloud, solutions that have gained traction due to major cloud vendors' recent implementations. Blockchain and IoT can be a perfect match due to the complementary characteristics they expose and the identified shortcomings that can be fixed.

Taking into consideration that each solution to a problem can give rise to new issues that need to be addressed we have to look forward to new developments in the Blockchain and internet of things disciplines.

\section{References}

[1] P. D.-I. K. Schwab, The Fourth Industrial Revolution, World Economic Forum, 2016. 
www.conferenceie.ase.ro

[2] N. Teslya and I. Ryabchikov, "Blockchain-Based Platform Architecture for Industrial IoT," in Proceedings of the XXth Conference of Open Innovations Association FRUCT. 562. 321-329., 2017.

[3] H. Sundmaeker, P. Guillemin, P. Friess and S. Woelfflé, Vision and Challenges for Realising the Internet of Things, Luxembourg: Publications Office of the European Union , 2010.

[4] K. Ashton, "Beginning the Internet of Things," 18 March 2016. [Online]. Available: https://medium.com/@kevin_ashton/beginning-the-internet-of-things-6d5ab6178801. [Accessed March 2019].

[5] "Definition of blockchain in English," Oxford University Press, 2019. [Online]. Available: https://en.oxforddictionaries.com. [Accessed March 2019].

[6] W. Viriyasitavat and D. Hoonsopon, "Blockchain characteristics and consensus in modern business processes," Journal of Industrial Information Integration, vol. 13, pp. 32-39, 2018.

[7] Cisco, "Fog Computing and the Internet of Things: Extend the Cloud to Where the Things Are," Cisco, 2015.

[8] M. Roberts, "Serverless Architectures," Thought Works, 22 May 2018. [Online]. Available: https://martinfowler.com/articles/serverless.html. [Accessed March 2019].

[9] M. Bestehorn and J. Metzner, "Serverless Industry 4.0 \& AI: Drive Business Insights from Machine Data," in Amazon Web Services Summit, Berlin, 2019.

[10] E. Alsaadi and A. Tubaishat, "Internet of Things: Features, Challenges and Vulnerabilities," International Journal of Advanced Computer Science and Information Technology (IJACSIT), vol. 4, pp. 1-13, 2015.

[11] I. Makhdoom, M. Abolhasan, H. Abbas and W. Ni, "Blockchain's adoption in IoT: The challenges, and a way forward," Journal of Network and Computer Applications, no. 125, pp. 251-279, 2019.

[12] J. Lin, W. Yu, N. Zhang, X. Yang, H. Zhang and W. Zhao, "A Survey on Internet of Things: Architecture, Enabling Technologies, Security and Privacy and Applications," IEEE Internet of Things Journal, vol. 4, p. 1125-1142, 2017.

[13] H. Atlam and G. B. Wills, "Intersections between IoT and distributed ledger," in Role of Blockchain Technology in IoT Applications, ELSEVIER, 2019.

[14] P. G. S. I. D. M. Panagiotis Radoglou Grammatikis, "Securing the Internet of Things: Challenges, threats and solutions," Internet of Things, no. 5, pp. 41-70, 2019.

[15] C.-F. Liao, C.-C. Hung and K. Chen, "Blockchain and the Internet of Things: A Software Architecture Perspective," in Business Transformation through Blockchain, Palgrave Macmillan, 2019.

[16] S. Hanselman, "How to Build a Kubernetes Cluster with ARM Raspberry Pi then run .NET Core on OpenFaas," 28 October 2017. [Online]. Available: https://www.hanselman.com/blog. [Accessed March 2018].

[17] A. Ellis, "OpenFaas," December 2016. [Online]. Available: https://www.openfaas.com/. [Accessed March 2019].

[18] X. Wang, X. Zha, W. Ni, R. P. Liu, J. Guo, X. Niu and K. Zheng, "Survey on blockchain for Internet of Things," Computer Communications, no. 136, pp. 10-29, 2019.

[19] D. Macrinici, C. Cartofeanu and S. Gao, "Smart contract applications within blockchain technology: A systematic mapping study," Telematics and Informatics, no. 35, pp. 23372354, 2018.

[20] A. Reyna, C. Martín, J. Chen, E. Soler and M. Díaz, "On blockchain and its integration with IoT. Challenges and opportunities," Future Generation Computer Systems, no. 88, pp. 173-190, 2018. 